\title{
Feasibility, Tolerability, Safety and Efficacy of a Pantothenic Acid Based Dietary Supplement in Subjects with Mild to Moderate Facial Acne Blemishes
}

\author{
Jillian L. Capodice* \\ Nutraceutical Medical Research, New York City, USA. \\ Email: ${ }^{*}$ jcapodice@nutraceuticalmedicalresearch.com \\ Received May $5^{\text {th }}, 2012$; revised June $8^{\text {th }}, 2012$; accepted June $20^{\text {th }}, 2012$
}

\begin{abstract}
Objective: It has been suggested that pantothenic acid may have antibacterial and skin softening activity. The aim of this study was to explore the feasibility, tolerability, safety and preliminary efficacy of oral administration of a dietary supplement containing pantothenic acid in healthy human males and females with mild to moderate facial acne vulgaris. Methods: An open-label, single arm study of healthy adults who had been previously diagnosed with mild to moderate acne vulgaris was performed. Subjects were asked to take the study agent, a dietary supplement containing pantothenic acid, for eight weeks. The primary endpoint of the study was to assess the feasibility of oral administration of the study agent in subjects over an eight week period. Safety and tolerability were measured utilizing the assessment of adverse events by the National Cancer Institute's Common Criteria for Adverse Event Reporting. Secondary endpoints measuring the efficacy of an oral panthothetic acid dietary supplement for the treatment of mild to moderate facial acne utilized changes in the extent of global facial skin blemishes, assessment of quality of life utilizing the Dermatology Life Quality Index (DLQI) and analysis of questions about the subject's beliefs and attitudes towards skin care and lifestyle. Results: Eleven subjects were enrolled and ten completed the study (90.9\%). There were no reported adverse events. Of the 10 evaluable subjects, the average age (mean $\pm \mathrm{SD}$ ) was $31.8 \pm 8$. Analysis of the global number of skin blemishes demonstrated a significant mean reduction in lesion count following the use of the study agent at week 8 (endpoint) $(11.18 \pm 6.38, \mathrm{p}=0.02)$ compared to the average number of baseline blemishes $(20.45 \pm 10.44)$. DLQI scores were significantly lower at week 8 vs. baseline $(p=0.0194)$. Conclusions: The results from this study indicated that the administration of a pantothenic based dietary supplement in healthy human adults with mild to moderate acne vulgaris is feasible, safe and well tolerated. Secondary analysis shows that administration of the study agent significantly reduced global facial blemishes. Further randomized, placebo-controlled trials are warranted.
\end{abstract}

Keywords: Pantothenic Acid; Dietary Supplement; Facial Blemishes; Acne

\section{Introduction}

Acne is a common disease of the hair follicles in the skin associated with an oil gland. Acne often appears in teens but can persist in up to two-thirds of adults [1,2]. Studies have shown that people with acne may suffer significant psychosocial burden and report poor quality of life [2]. The mainstays of treatment include over-the-counter and prescription pharmaceutical drugs including oral and topical antibiotics and retinoids and oral contraceptives [3]. Other procedures include lasers, phototherapy and other light sources [3]. Recently, approaches from complementary and alternative medicine (CAM) including the use of natural products including topical agents containing vitamin or botanical ingredients, dietary interventions

"Corresponding author. and acupuncture have been examined [3,4]. Some agents may have clear mechanisms of action such as vitamins and dietary supplements like pyridoxine or omega-3 fatty acids however there is limited data on the use of dietary supplements in the treatment of dermatologic conditions such as acne [4]. A recent study found that subjects with acne were more apt to use CAM based on lower risk, perceived safety of natural agents, holistic attitudes towards health and improved quality of life, however prospective analysis of various practices and procedures are lacking and need to be performed in order to determine feasibility, tolerability and efficacy of these approaches [4,5]. Given this background we chose to examine the feasibility, tolerability, and safety of a novel dietary supplement containing pantothenic acid in adult males and females with mild to moderate skin blemishes. Secon- 
dary aims were to measure changes in the extent of global facial skin lesions and quality of life.

\section{Materials and Methods}

\subsection{Subjects}

Healthy human male and female volunteers over the age of 18 , with a previously diagnosed history of acne vulgaris, more than 10 facial blemishes of any type, able to understand and sign the informed consent in the English language and in good health were eligible for the study. Excluded from the study were subjects taking any oral or topical prescription drug for acne vulgaris, subjects currently undergoing treatment for acne vulgaris besides daily hygienic skin care, known renal or hepatic impairment, scheduled elective surgery or other procedures requiring general anaesthesia during the study, participation in another research study involving an investigational product in the past month, hypersensitivity or known allergy to any ingredients in the study agent, current use of any dietary supplement for skin health (besides a multivitamin), history or presence of gastric ulcer or duodenal ulcer, history of any gynaecologic condition, history of any psychiatric disorder and current smoking or smoking within the past month. The protocol for this study was conducted under Good Clinical Practice in concordance with the Declaration of Helinski and it's amendments.

\subsection{Study Procedures}

The study was performed between December 2011 and April 2012. Consecutive human volunteers that met the eligibility criteria were instructed to read, understand and sign the written informed consent and model release disclaimer. At baseline, subjects were asked to complete demographic information, have a photograph taken of their face, complete the Dermatology Life Quality Index (DLQI), and other health history information. The DLQI is a general questionnaire that evaluates quality of life (QOL) in dermatology patients and consists of ten questions about symptoms, feelings, daily activities, type of clothing, social or physical activities, exercise, job or education, interpersonal relationships, marriage relationships, and treatment. Higher scores indicate a poorer quality of life [6]. Subjects were then assigned to take the study agent, the dietary supplement, Pantothen (Pantothen $^{\text {TM }}$, Avilan Marketing LLC, New York, NY), two tablets twice a day with food, for eight weeks. Each 4 tablet dose of Pantothen contain the following ingredients: thiamine- $1.5 \mathrm{mg}$, riboflavin $-1.7 \mathrm{mg}$, niacin -20 $\mathrm{mg}$, pyridoxine $-2 \mathrm{mg}$, folic acid- $400 \mathrm{mgc}$, cyanocobalamin $-6 \mathrm{mcg}$, biotin $-300 \mathrm{mcg}$, pantothenic acid$2.2 \mathrm{~g}$ and L-carnitine-733.3 mg. At weeks 4 (mid-point) and week 8 (end-point) volunteers were asked about their compliance in taking the study agent and any side effects and tolerability as measured by the National Cancer Institute's Common Toxicity Criteria for Adverse Events 3.0 (NCI CTCAE). Subjects were also asked to complete the DLQI and have a photograph of their face taken. At the final visit overall satisfaction with the study agent was assessed using a 5-point scale: $2=$ marked improvement, $1=$ slight improvement, $0=$ unchanged, $-1=$ worsening, $-2=$ marked worsening.

\subsection{Statistics}

The primary feasibility outcome was number of subjects enrolled versus those completing the study defined as $80 \%$ of the volunteers completing the 4 -week visit and/or $70 \%$ of volunteers completing all 8 weeks of consuming the study agent. The tolerability and safety outcome was the incidence of adverse effects, complication/illness and/ or serious medical events due to the study agent as measured by the NCI CTCAE. The secondary efficacy outcome of this study was change in the number of global facial blemishes from baseline to week 8 as measured by lesion count. Other outcomes were scores on the DLQI. The number of facial blemishes and DLQI scores were measured by a paired t-test comparing counts/ scores at baseline (week 0) to week 8. Descriptive analyses were performed for demographics utilizing characteristic measures such as mean, standard deviation, and range.

\section{Results}

\subsection{Subjects and Feasibility}

In total 13 subjects were screened, 11 subjects were enrolled and 10 completed the study. The 8-week completion rate $(90.0 \%)$ met the feasibility endpoint. Of the 10 evaluable subjects the mean \pm SD age was $31.8 \pm 8.4$. Demographics and baseline characteristics are summarized in Table 1. The reasons for premature withdrawal were lost to follow up [1].

\subsection{Safety and Tolerability Outcomes}

No serious side effects were reported throughout the study and there were no reported adverse events.

\subsection{Facial Blemishes}

The area of the face that was most affected in the study population was the cheek, followed by the forehead and chin (60 vs. 20 vs. 20 percent respectively). Analysis of the number of facial skin blemishes (global acne count) demonstrated a significant mean reduction in lesion count from baseline (lesion count $20.45 \pm 10.44$ ) to week 
Table 1. Demographics.

\begin{tabular}{cc}
\hline Characteristic & Frequency \\
\hline N & 10 \\
Mean age, years \pm SD & $31.8 \pm 8.4$ \\
Sex, no (\%) & \\
Male & $2(20 \%)$ \\
Female & $8(80 \%)$ \\
Race, no (\%) & \\
White & $5(50 \%)$ \\
Black & $2(20 \%)$ \\
Latino & $2(10 \%)$ \\
Asian & $1(10 \%)$ \\
BMI (mean, SD) & $24.3 \pm 3.8$ \\
Baseline global facial lesion & $20.45 \pm 10.44$ \\
Area most predominately affected & \\
Cheek & $6(60 \%)$ \\
Forehead & $2(20 \%)$ \\
Chin & $2(20 \%)$ \\
\hline
\end{tabular}

8 lesion count $(11.18 \pm 6.38, \mathrm{p}=0.02)$ (Figure 1(a)). Figure 1(b) shows a subject at baseline versus week 8 reduction in global acne count in a study participant.

\subsection{Dermatology Life Quality Index and Patient Reported Outcomes}

The mean \pm SD DLQI score at baseline vs. week 8 was $9.73 \pm 4.2$ vs. $6.09 \pm 2.21, p=0.0194 .6$ of $10(60 \%)$ of volunteers reported marked improvement, 3 of $10(30 \%)$ reported slight improvement and only 1 of $10(10 \%)$ reported no change in overall subject satisfaction.

\section{Discussion}

Pantothenic acid (vitamin B5) is a water-soluble B-complex vitamin that has may have beneficial effects on skin health. The results of this study demonstrated it was both feasible and safe for healthy human volunteers with known mild to moderate acne to take a dietary supplement (Pantothen ${ }^{\mathrm{TM}}$ ) containing pantothenic acid. Our results also showed that there was a greater than $56 \%$ reduction in the number of global facial blemishes after 8 weeks of Pantothen supplementation. Significantly lower scores on the Dermatology Life Quality Index corresponding to improved quality of life were also found in subjects taking the study agent.

It has been suggested that pantothenic acid and some

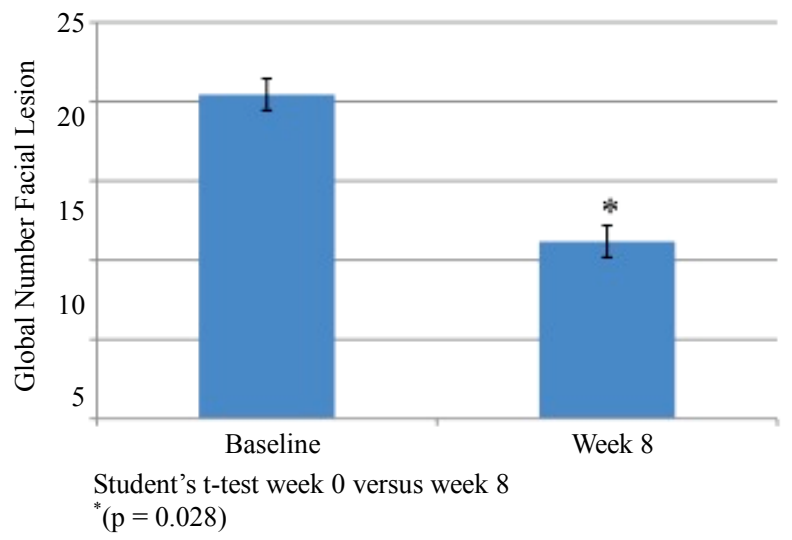

(a)

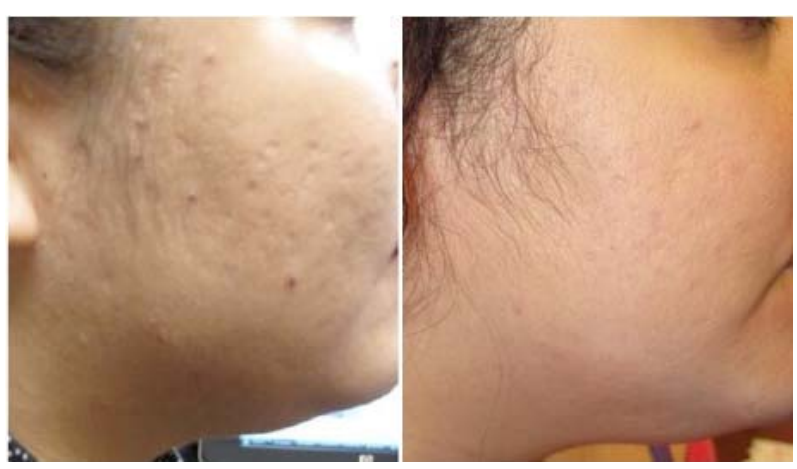

(b)

Figure 1. (a) Frequency of facial blemishes; (b) Clinical photograph at baseline vs. week 8 .

of its analogs may have antibacterial and skin softening activity. Pantothenic acid is converted into 4'-phosphopantetheine which is then converted to co-enzyme A (CoA) utilizing adenosine tri-phosphate (ATP) [7-9]. $\mathrm{CoA}$ is involved in lipid metabolism and many other cellular processes and it has been shown that pantothenic acid may regulate epidermal barrier function through proliferation and differentiation of keratinocytes via CoA metabolism [10]. It is possible that the reduction in the amount of global skin lesions in volunteers following oral administration of the pantothenic acid based study agent may function through these mechanisms. However the exact mechanism of this effect is not understood.

The bioavailability of pantothenic acid has been reported in the range of $40-63$ percent and amounts found in 24-hour urine samples have been shown to correlate with intake [11]. However in the adult with acne, little is known about the role of co-enzyme A and other factors which may cause acne including relationships to sebum production, keritinzation alteration and/or effect on inflammatory modulators [12]. While it is uncommon for adults to suffer from clinically significant pantothenic acid deficiency, it has been postulated that low levels and/or dysreuglation of fatty acid metabolism due to 
pantothenic acid deficiency may be a factor in acne pathogenesis [13]. Even though pantothenic acid is the main ingredient in Pantothen, there are a number of other vitamins that may contribute to a synergistic mechanism of action. This could include the B-complex vitamins and/ or L-carnitine which has been recently shown to reduce sebum secretion in a human sebaceous cell line [14].

This study also demonstrated improved quality of life in subjects with facial blemishes as measured by the Dermatology Life Quality Index. It has been well demonstrated that adults with acne have reported reduced quality of life with regard to dissatisfaction and patient bother [2]. Moreover, it has been suggested that all studies of acne include assessments of quality of life because such assessements appear to be important correlates in measuring the success of treatment [15].

The limitations of this study are that we tested feasibility in subjects with heterogeneous lesions, had a small sample size and no control group. Due to these factors our exploratory findings cannot be directly attributed to the administration of the study agent as it is possible that volunteers improved because they made lifestyle or other changes that were not reported in their follow up visits.

Strengths of our study include pilot analysis of feasibility and safety that will enable us to better design and power a larger study and examine potential mechanisms of action of the study agent and dosage. Given the feasibility, tolerability, safety and positive trend in improving symptoms and quality of life, a larger randomized, placebo controlled study of Pantothen is warranted.

\section{REFERENCES}

[1] S. Knutsen-Larson, A. L. Dawson, C. A. Dunnick and R. P. Dellavalle, "Acne Vulgaris: Pathogenesis, Treatment, and Needs Assessment," Dermatologic Clinics, Vol. 30, No. 1, 2012, pp. 99-106, viii-ix. doi:10.1016/j.det.2011.09.001

[2] L. E. Barnes, M. M. Levender, A. B. Fleischer Jr. and S. R. Feldman, "Quality of Life Measures for Acne Patients," Dermatologic Clinics, Vol. 30, No. 2, 2012, pp. 293-300, ix. doi:10.1016/j.det.2011.11.001

[3] H. C. Williams, R. P. Dellavalle and S. Garner, "Acne Vulgaris," The Lancet, Vol. 379, No. 9813, 2012, pp. 361-372. doi:10.1016/S0140-6736(11)60321-8

[4] P. J. Magin, J. Adams, G. S. Heading, D. C. Pond and W. Smith, "Complementary and Alternative Medicine Therapies in Acne, Psoriasis, and Atopic Eczema: Results of a Qualitative Study of Patients' Experiences and Perceptions," Journal of Alternative and Complementary Medicine, Vol. 12, No. 5, 2006, pp. 451-457. doi:10.1089/acm.2006.12.451
[5] Z. El-Akawi, N. Abdel-Latif and K. Abdul-Razzak, "Does the Plasma Level of Vitamins A and E Affect Acne Condition?" Clinical and Experimental Dermatology, Vol. 31, No. 3, 2006, p. 430. doi:10.1111/j.1365-2230.2006.02106.x

[6] A. Y. Finlay and G. K. Khan, "Dermatology Life Quality Index (DLQI) - A Simple Practical Measure for Routine Clinical Use," Clinical and Experimental Dermatology, Vol. 19, No. 3, 1994, pp. 210-216. doi:10.1111/j.1365-2230.1994.tb01167.x

[7] F. B. Camargo Jr., L. R. Gaspar and P. M. Maia Campos, "Skin Moisturizing Effects of Panthenol-Based Formulations," Journal of Cosmetic Science, Vol. 62, No. 4, 2011, pp. 361-370.

[8] H. R. Jerajani, H. Mizoguchi, J. Li, D. J. Whittenbarger and M. J. Marmor, "The Effects of a Daily Facial Lotion Containing Vitamins B3 and E and Provitamin B5 on the Facial Skin of Indian Women: A Randomized, DoubleBlind Trial," Indian Journal of Dermatology, Venereology and Leprology, Vol. 76, No. 1, 2010, pp. 20-26. doi:10.4103/0378-6323.58674

[9] G. S. Kelly, "Pantothenic Acid. Monograph," Alternative Medicine Review, Vol. 16, No. 3, 2011, pp. 263-274.

[10] D. Kobayashi, M. Kusama, M. Onda and N. Nakahata, "The Effect of Pantothenic Acid Deficiency on Keratinocyte Proliferation and the Synthesis of Keratinocyte Growth Factor and Collagen in Fibroblasts," Journal of Pharmacological Sciences, Vol. 115, No. 2, 2011, pp. 230234. doi:10.1254/jphs.10224SC

[11] B. R. Eissenstat, B. W. Wyse and R. G. Hansen, "Pantothenic Acid Status of Adolescents," The American Journal of Clinical Nutrition, Vol. 44, No. 6, 1986, pp. 931-937.

[12] H. P. Gollnick, A. Y. Finlay and N. Shear, "Global Alliance to Improve Outcomes in Acne. Can we Define Acne as a Chronic Disease? If So, How and When?" American Journal of Clinical Dermatology, Vol. 9, No. 5, 2008, pp. 279-284. doi:10.2165/00128071-200809050-00001

[13] L. H. Leung, "Pantothenic Acid Deficiency as the Pathogenesis of Acne Vulgaris," Medical Hypotheses, Vol. 44, No. 6, 1995, pp. 490-492. doi:10.1016/0306-9877(95)90512-X

[14] R. I. Peirano, T. Hamann, H. J. Düsing, M. Akhiani, U. Koop, T. Schmidt-Rose and H. Wenck, "Topically Applied L-Carnitine Effectively Reduces Sebum Secretion in Human Skin," Journal of Cosmetic Dermatology, Vol. 11, No. 1, 2012, pp. 30-36. doi:10.1111/j.1473-2165.2011.00597.x

[15] J. R. Ingram, D. J. Grindlay and H. C. Williams, "Management of Acne Vulgaris: An Evidence-Based Update," Clinical and Experimental Dermatology, Vol. 35, No. 4, 2010, pp. 351-354. doi:10.1111/j.1365-2230.2009.03683.x 